\title{
A short ode to jackknife empirical likelihood procedures
}

\section{Editorial}

Empirical likelihood (EL) is a statistical approach for nonparametric inference. The classical empirical likelihood (EL) was proposed by Thomas and Grunkemeier, ${ }^{1}$ who inverted a nonparametric likelihood ratio test to obtain confidence intervals for the survival probability for right censored data. In empirical likelihood (EL) theory, an unknown parameter vector can be estimated by maximizing the empirical likelihood, under constraints. Based on the idea, $\mathrm{Owen}^{2}$ developed the empirical likelihood (EL) method. By using EL method, researchers can determine the shape of confidence regions without having to pick a parametric family for the data. A number of researchers have implemented this method, for example, Zhao and $\mathrm{Chen}^{3}$ made empirical likelihood inference for censored median regression models via nonparametric kernel estimation, and the linear transformation model with interval-censored failure time data was studied in Zhang and Zhao. ${ }^{4}$ It is noticeable that EL has been recognized as a useful tool in statistical sciences.

Although empirical likelihood approach shows attractive properties, in practice, the computational cost might be expensive when dealing with more complicated problems. A simpler and more computationally reliable method, jackknife empirical likelihood (JEL), has been widely used. JEL method is proposed by Jing et al. ${ }^{5}$ which combines EL and jackknife resampling method. JEL constructs confidence region by introducing jackknife pseudo-values into the EL method. A major advantage of the JEL method is its simplicity. Moreover, JEL is very appealing because it is more general than usual parametric likelihood as it can be applied to test complicated hypotheses. For instance, Zhao et al. ${ }^{6}$ proposed using JEL to study the mean absolute deviation, and Lin et al. ${ }^{7}$ developed JEL for the error variance in a linear regression model.

Furthermore, JEL is commonly used in survival analysis. It not only provides efficient evaluation over survival functions regardless of complete or censored data, but also can be applied in different models, such as accelerated failure time models (Bouadoumou et al. ${ }^{8}$ ) and linear transformation models (Yang et al. ${ }^{9}$ ). JEL has other applications in clinical experiments, for example, receiver operating characteristic (ROC) curve, a widely used graphical plot evaluating the discriminating power of a diagnostic test. Liu and $\mathrm{Zhao}^{10}$ proposed semi-empirical likelihood based confidence intervals for ROC curves of two populations with missing data. It is then extended to the difference of two volumes under ROC surfaces (An and Zhao ${ }^{11}$ ). Yang and Zhao ${ }^{12}$ constructed smoothed jackknife EL confidence intervals for the difference of ROC curves. Yang and Zhao ${ }^{13}$ made smoothed JEL inference for ROC curves with missing data. Furthermore, Yang et al. ${ }^{14}$ made JEL inference for the partial area under ROC curves. In addition, there are more research works about difference of quantiles and difference of two Gini indices, like Wang and Zhao, ${ }^{15}$ Yang and Zhao, ${ }^{16}$ Yang and Zhao. ${ }^{17}$ These research studies indicate that the JEL based methods are employed in many bio statistical fields, and it can be handy in dealing with more general statistics beyond classical U-statistics. Notably, JEL methods can be easily implemented in a standard software environment, and it will ease the computational burden for practical use.
Volume 7 Issue I - 2018

\author{
Xue Yu, Yichuan Zhao \\ Department of Mathematics and Statistics, Georgia State \\ University, USA
}

Correspondence: Yichuan Zhao, Department of Mathematics and Statistics, Georgia State University, Atlanta, GA 30303, USA, Email yichuan@gsu.edu

Received: January 4, 20I8 | Published: January 08, 2018

\section{Acknowledgement}

None

\section{Conflict of interest}

None

\section{References}

1. Thomas DR, Grunkemeier GL. Confidence interval estimation of sur $\neg$ vival probabilities for censored data. Journal of the American Statistical Association. 1975;70(352):865-871.

2. Owen AB. Empirical likelihood ratio confidence intervals for a single functional. Biometrika. 1988;75(2):237-249.

3. Zhao Y, Chen F. Empirical likelihood inference for censored median regression model via nonparametric kernel estimation. Journal of Multivariate Analysis. 2008;99(2):215-231.

4. Zhang Z, Zhao Y. Empirical likelihood for linear transformation models with interval-censored failure time data. Journal of Multivariate Analysis. 2013;116:398-409.

5. Jing BY, Yuan J, Zhou W. Jackknife empirical likelihood. Journal of the American Statistical Association. 2009;104(487):1224-1232.

6. Zhao Y, Meng X, Yang H. Jackknife empirical likelihood inference for the mean absolute deviation. Computational Statistics \& Data Analysis. 2015;91:92-101.

7. Lin H-L, Li Z, Wang D, et al. Jackknife empirical likelihood for the error variance in linear models. Journal of Nonparametric Statistics. 2017;29(2):151-166.

8. Bouadoumou M, Zhao Y, Lu Y. Jackknife empirical likelihood for the accelerated failure time model with censored data. Communications in Statistics-Simulation and Computation. 2015;44(7):1818-1832.

9. Yang H, Liu S, Zhao Y. Jackknife empirical likelihood for linear transformation models with right censoring. Annals of the Institute of Statistical Mathematics. 2016;68(5):1095-1109.

10. Liu X, Zhao Y. Semi-empirical likelihood inference for the ROC curve with missing data. Journal of Statistical Planning and Inference. 2012;142(12):3123-3133.

11. An Y, Zhao Y. Jackknife empirical likelihood for the difference of two volumes under ROC surfaces. Annals of the Institute of Statistical Mathematics, In press: 2017;1-18. 
12. Yang H, Zhao Y. Smoothed jackknife empirical likelihood inference for the difference of ROC curves. Journal of Multivariate Analysis. 2013;115:270-284.

13. Yang H, Zhao Y. Smoothed jackknife empirical likelihood inference for ROC curves with missing data. Journal of Multivariate Analysis. 2015;140(C):123-138.

14. Yang H, Lu K, Zhao Y. A nonparametric approach for partial areas under ROC curves and ordinal dominance curves. Statistica Sinica. 2017;27:357-371.
15. Wang D, Zhao Y. Jackknife empirical likelihood for comparing two Gini indices. Canadian Journal of Statistics. 2016;44(1):102-119.

16. Yang H, Zhao Y. Smoothed jackknife empirical likelihood for the difference of two quantiles. Annals of the Institute of Statistical Mathematics. 2017;69(5):1059-1073.

17. Yang H, Zhao Y. Smoothed jackknife empirical likelihood for the one-sample difference of quantiles. Computational Statistics \& Data Analysis. 2018;120:58-69. 